\title{
Investigative radiochemistry - a key element in nuclear forensics
}

\author{
By K. Mayer*, M. Wallenius, Z. Varga, T. Wiss and Th. Fanghänel \\ European Commission, Joint Research Center, Institute for Transuranium Elements, Karlsruhe, Germany
}

(Received December 22, 2009; accepted in revised form November 12, 2010)

\section{Nuclear forensics / Uranium / Age dating / Heisenberg}

\begin{abstract}
Summary. Since the fall of the Iron Curtain illicit trafficking of nuclear and radioactive material has become an issue of concern both on the political and the scientific level. Seized material may be analysed in order to obtain clues on its origin and intended use and to prevent diversion of material from the same source in the future. Nuclear materials (uranium or plutonium) are of particular worry due to the nuclear proliferation risk associated with the material. Nuclear forensic investigations are aimed at the fact that nuclear material carries (inherent) information on its history, including on its origin and the processes applied for its production. Important conclusions can be drawn from decay products, activation products and fission products. Chemical impurities and the isotopic composition of certain major and minor constituents may provide additional information. Comparison of the measured results with nuclear material databases may yield evidence on the production site. The paper will describe the methodologies developed for addressing the above issues, focussing on radiochemical methods. Examples of nuclear forensic casework will illustrate the experience gathered in these areas.
\end{abstract}

\section{Introduction}

Nuclear material is subject to strict international controls to prevent nuclear proliferation. Therefore the interception of nuclear material from illicit activities trigger questions on the history of the material, i.e. its place and time of production, the route it has taken and the intended use. Nuclear forensics is a methodology that can provide results for answering these questions. Nuclear forensics makes use of the information inherent to the material, aiming at establishing a relation between the sample under investigation, the origin of the material and the place of theft or diversion (i.e. the place where the material was taken out of regulatory control). Having identified the latter, reinforced preventive measures can be implemented in order to minimize the risk of future thefts or diversions. Thus, nuclear forensics supports non-proliferation. This information may also be of relevance for prosecution in case of criminal or unauthorized acts involving nuclear material.

\footnotetext{
*Author for correspondence

(E-mail: Klaus.MAYER@ec.europa.eu).
}

\section{Nuclear forensics methodology}

Nuclear forensics investigations require a systematic and scientifically sound approach. In consequence, the methodologies in place or under development for acquiring measurement results have to be efficient and enable timely conclusions in order to be useful. Moreover, the conclusions that are provided by nuclear forensics laboratories must be credible and not speculative. Measurement data are solid facts, provided they were obtained with properly validated methods and their uncertainties have been carefully evaluated. Parameters may be source material inherited or process inherited. Process related parameters may arise from chemical operations, from technical operations or from physical operations (including nuclear reactions and radioactive decay). This leads to a complex pattern of signatures and of course to analytical noise, i.e. data that cannot be usefully related to the material history. Parameters such as the isotope ratios of the nuclear material, the chemical impurities, certain stable isotope ratios, non-metallic impurities, structural aspects or decay products are signatures that contribute to the overall pattern. The complexity of these signatures obviously leads to an analytical and interpretational challenge. The multiple and complementary parameters measured in the nuclear forensics investigations, however, offer opportunities for signatures development and thus for credible interpretation (see Table 1).

Table 1. Characteristic parameters to be measured on nuclear maerial of unknown origin and method for comparative evaluation of data.

\begin{tabular}{ll}
\hline Parameter & Comparison against \\
\hline U isotope ratios & Data base \\
Pu isotope ratios & Model calculations \\
& $\begin{array}{l}\text { Data base } \\
\text { Metallic impurities }\end{array}$ \\
(concentrations, patterns) & Pacess knowledge \\
Stable isotope ratios & Data base, known samples \\
Macroscopic appearance & Process knowledge \\
& Database, known samples \\
Microscopic appearance & Process knowledge \\
Radioisotopes & Database, known samples \\
Non-metallic impurities & Model calculations \\
& Process knowledge \\
\hline
\end{tabular}


The majority of these signatures can best be determined by radiochemical methods. Thus, radiochemical analysis forms the backbone of nuclear forensic investigations.

The measurement and interpretation process should be fully transparent to the end-user of the information. It is therefore important to include nuclear forensics considerations throughout the entire incident response process. It requires close consideration at the conceptual level (i.e. include nuclear forensics in the national response plan), at the operational level (i.e. include appropriate sampling procedures in the crime scene management) and at the analytical level (i.e. include quality control mechanisms in the laboratory procedures).

\section{Case studies: uranium metal}

The methodology applied in nuclear forensic investigations shall be illustrated using two examples. Particular attention will be given to the radiochemical methods used for obtaining information on the history of the samples.

The first sample, a uranium metal cube was presented to ITU in 2002 for nuclear forensic investigations. The cube was said to date back to the German nuclear program conducted in World War II under the scientific leadership of Werner Heisenberg. It will henceforth be referred to as "Heisenberg Cube" (Fig. 1).

Two years later, scientists of the Max-Plack Institute for Nuclear Physics in Heidelberg discovered during a clean-up operation a uranium metal plate in an old safe. The material proved to be of natural isotopic composition and showed a total mass some $2 \mathrm{~kg}$. The plate was attributed to Karl Wirtz, a physicist who had worked with Heisenberg on the nuclear program in the 1940's. According to its mass and geometry, it might have been used in the reactor experiments based on the alternating layer approach. It will henceforth be referred to as "Wirtz Plate" (Fig. 2).

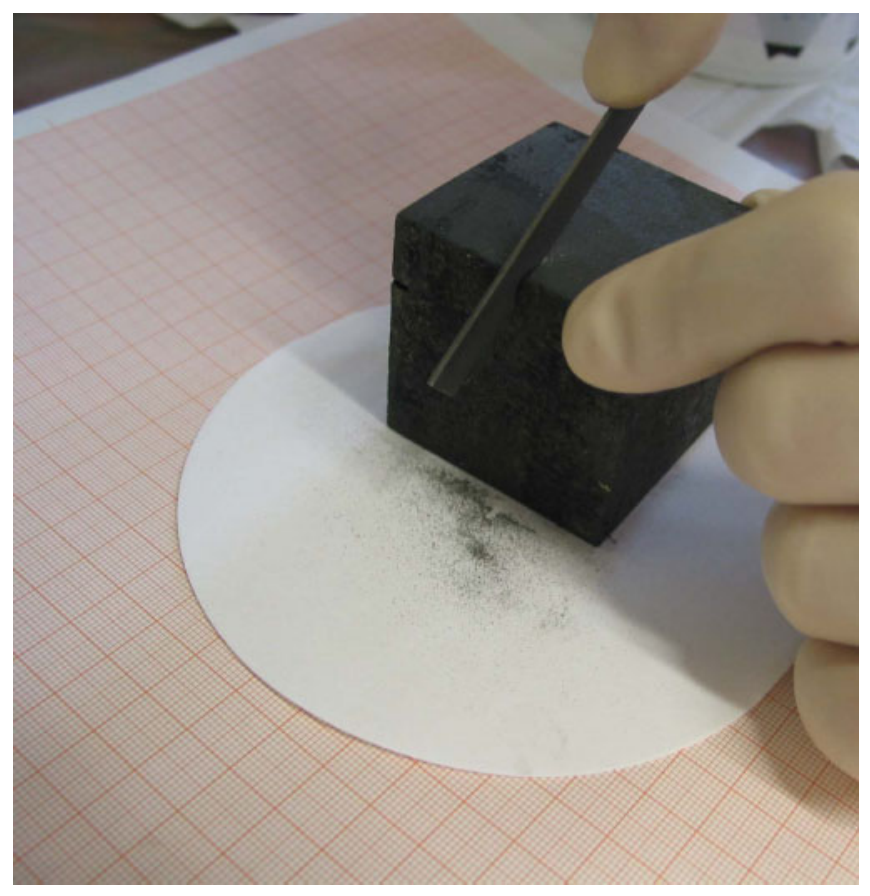

Fig. 1. "Heisenberg Cube".

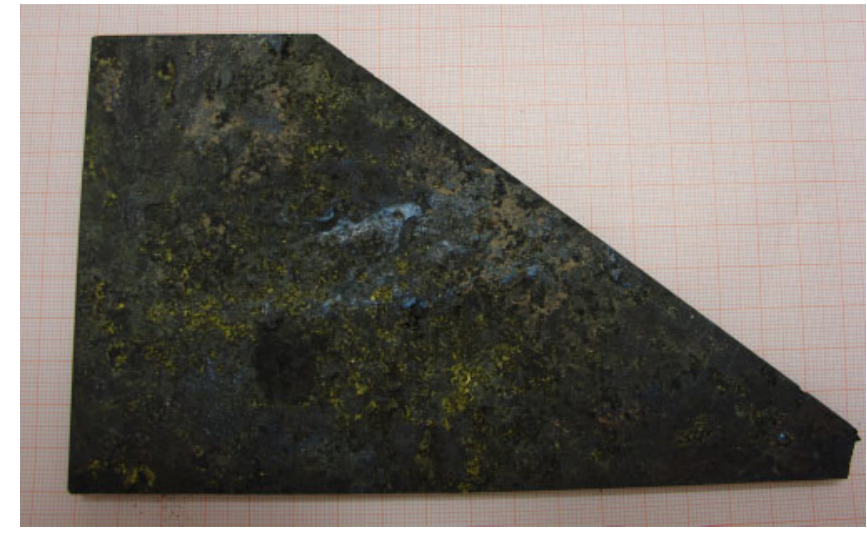

Fig. 2. "Wirtz Plate".

Nuclear forensic investigations were carried out in order to verify the authenticity of the two materials and to tell as much as possible about the history and the origin of the samples. Some of the results obtained from these two materials are presented and discussed hereafter, aiming at illustrating the wealth of information that can be obtained through investigative radiochemistry. These investigations comprised the analysis of metallic impurity elements, a dedicated investigation of the rare-earth element pattern in the samples, the isotopic composition of the uranium, the strontium isotopic composition and the determination of the latest chemical purification of the material. Table 2 shows the analytical scheme applied to the two materials.

\section{Uranium isotope ratio measurements}

Uranium isotope measurements were carried out by multicollector inductively coupled plasma mass spectrometry (MC-ICP-MS) and by thermal ionisation mass spectrometry (TIMS) using a Triton instrument (Thermo Corporation). For the TIMS measurements a double filament assembly was used and the instrument was run in a modified total evaporation mode [1], thus, the ion currents of all isotopes were recorded simultaneously and the signals were integrated over the entire measurement time. This eliminates time dependent isotope fractionation effects. The ${ }^{234} \mathrm{U}$ and ${ }^{236} \mathrm{U}$ ion currents were recorded using micro channeltrons (continuous dynode electron multiplier), while Faraday Cups were used for measuring the signals arising from ${ }^{235} \mathrm{U}$ and ${ }^{238} \mathrm{U}$ ions.

In neither of the two samples was a ${ }^{236} \mathrm{U}$ ion current above the detection limit recorded. The detection limit is mainly determined by the abundance sensitivity of the mass

Table 2. Isotope abundance ratios measured by thermal ionisation mass spectrometry. Values in brackets indicate the measurement uncertainty, expressed as combined standard uncertainty $U_{\mathrm{c}}$ with a coverage factor $k=2$.

\begin{tabular}{lccc}
\hline & $n\left({ }^{234} \mathrm{U}\right) / n\left({ }^{238} \mathrm{U}\right)$ & $n\left({ }^{235} \mathrm{U}\right) / n\left({ }^{238} \mathrm{U}\right)$ & $n\left({ }^{236} \mathrm{U}\right) / n\left({ }^{238} \mathrm{U}\right)$ \\
\hline Wirtz Plate & $\begin{array}{c}0.00005472 \\
(12)\end{array}$ & $\begin{array}{c}0.00725421 \\
(70)\end{array}$ & $\leq 0.00000000013$ \\
& 0.00005480 & 0.00725356 & $\leq 0.00000000020$ \\
Heisenberg & $(15)$ & $(70)$ & $(18)$ \\
Cube & & & \\
\hline
\end{tabular}




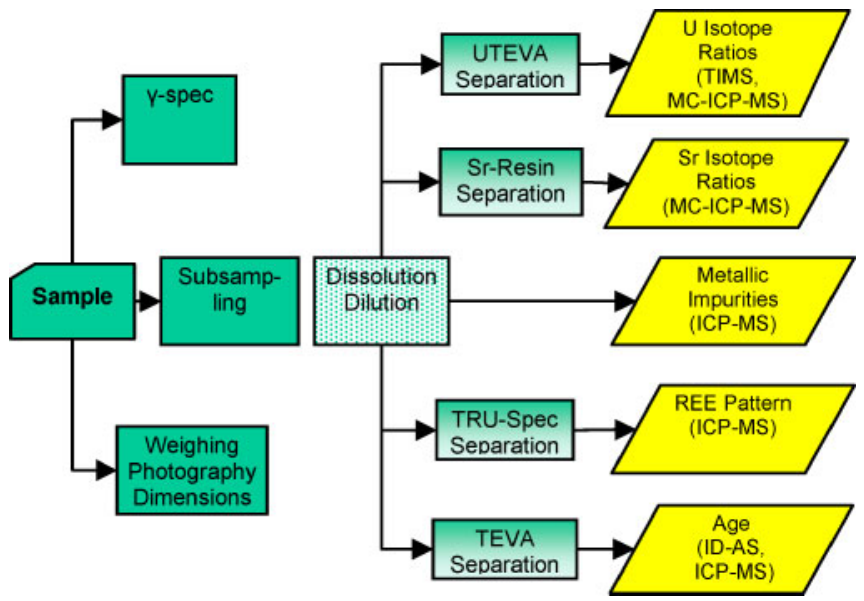

Fig. 3. Analytical scheme applied to the two uranium samples, underlining the role of radiochemical methods in nuclear forensics.

spectrometer used. Further investigations using accelerator mass spectrometry are being carried out (at Australian National University). The $n\left({ }^{235} \mathrm{U}\right) / n\left({ }^{238} \mathrm{U}\right)$ isotope abundance ratio in the two samples agrees very well. Similarly, no significant difference in the $n\left({ }^{234} \mathrm{U}\right) / n\left({ }^{238} \mathrm{U}\right)$ ratio was observed. Thus, the uranium isotopic composition did not provide any evidence of an exposure of the uranium to a significant neutron dose, that would have yielded in the production of small amounts of ${ }^{236} \mathrm{U}$ (due to neutron capture by ${ }^{235} \mathrm{U}$ ). The good between the isotope ratios of the samples does not suggest the materials are of different origin. As a single parameter, however, the uranium isotopic composition cannot be considered as a proof of an identical origin of the two materials.

\section{Rare Earth elemental pattern}

An aliquot of the dissolved sample (containing approximately $10 \mathrm{mg}$ of uranium) was taken for a group separation of the rare-earth elements (REE) from the uranium matrix. The method is described in detail elsewhere [2]. To summarise, the REE content of the sample aliquots were separated using extraction chromatography by the selective retention of trivalent REEs on the TRU ${ }^{\mathrm{TM}}$ resin in $3 \mathrm{M}^{\mathrm{ni}}$ tric acid medium. After washing the column and removal of the non-retained matrix components, the REE's were stripped from the column using $\mathrm{HCl}$. The method was optimized to avoid any chemical fractionation of the REE's during the separation and to achieve high selectivity over uranium. After the addition of $200 \mu$ ultra-pure $\mathrm{HNO}_{3}$ to the final fractions, the samples were evaporated to almost complete dryness on a hot-plate in order to destroy any organic resin residue. The residue was dissolved in $1 \mathrm{ml}$ of $2 \mathrm{~m} / \mathrm{m} \%$ ultra-pure nitric acid while heating slightly. After the gravimetric weighing of the final fractions and the addition of $\mathrm{Rh}$ internal standard, the samples were analysed by ICP-SFMS using external calibration. Using this separation method, the detection limits could be improved by approximately two orders of magnitude compared to a direct measurement. Due to the chemical separation, there was no need to use corrections for the spectral interferences (e.g. for oxides) and also serious matrix effects could be avoided. The mass spectrometric analysis was carried out using a double-focusing magnetic sector inductively coupled plasma mass spectrometer equipped with a single electron multiplier (ELEMENT2, Thermo Electron Corp., Bremen, Germany). All measurements were carried out in low resolution mode $(R=300)$ using a low-flow microconcentric nebulizer operated in a self-aspirating mode (flow rate was approximately $100 \mu \mathrm{L} \mathrm{min}^{-1}$ ). The REE concentrations are normalized to the chondrite values of Anders and Grevesse [3].

As can be seen from Fig. 4, the REE patterns of the "Heisenberg Cube" and of the "Wirtz Plate" are very similar. The measurement results of two comparison samples are also given on that graph. Up to the 1940's there were very few applications for uranium and in consequence uranium was mined only at very few places around the world. The Germans had at the time access to uranium from Belgian Congo and from Joachimstal (today: Jachymov, Czech Republic). These two uranium deposits are from different geological formation types. The Joachimstal ore is a quartzpebble conglomerate deposit and shows a relatively flat REE pattern, with depletion in the heavier REE's and a negative $\mathrm{Eu}$ anomaly. In contrast to that, the ore from Belgian Congo (Shinkolobwe) is an unconformity-related uranium ore, and has a bell-shaped REE pattern. In conclusion, the REE patterns exclude Belgian Congo as origin of the uranium for the production of the two uranium metal samples. The REE patterns suggest that both metals were produced

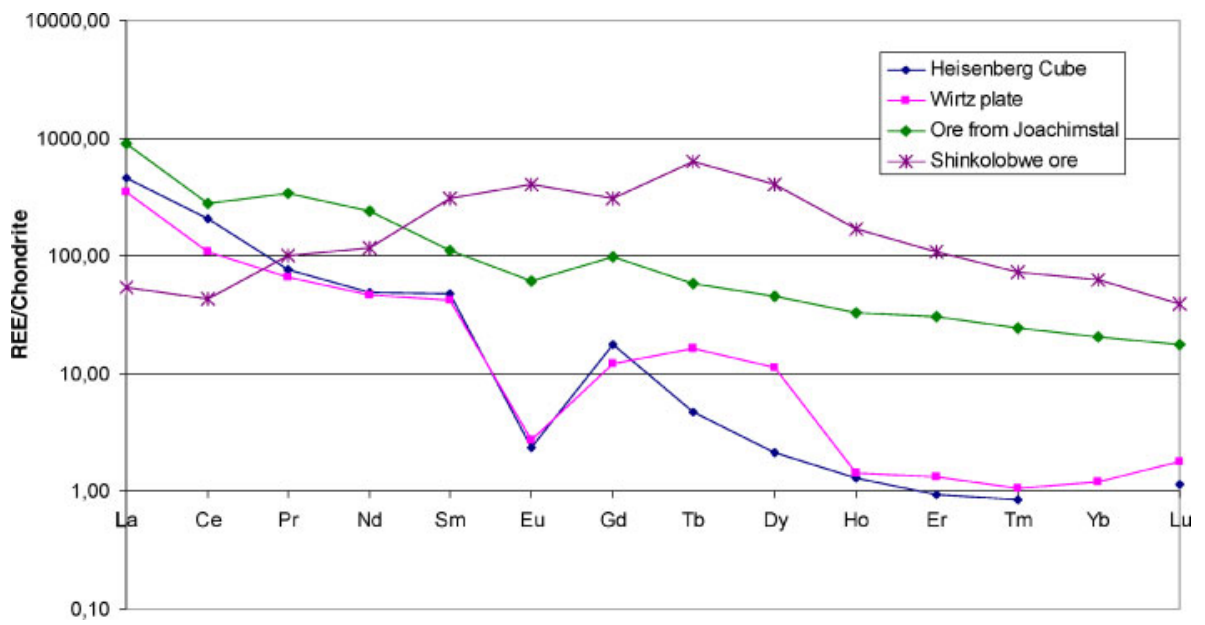

Fig. 4. Pattern of the rare earth elements, normalized to chondrite. The graph clearly indicates a high similarity of the patterns of the "Heisenberg Cube" and the "Wirtz Plate". 


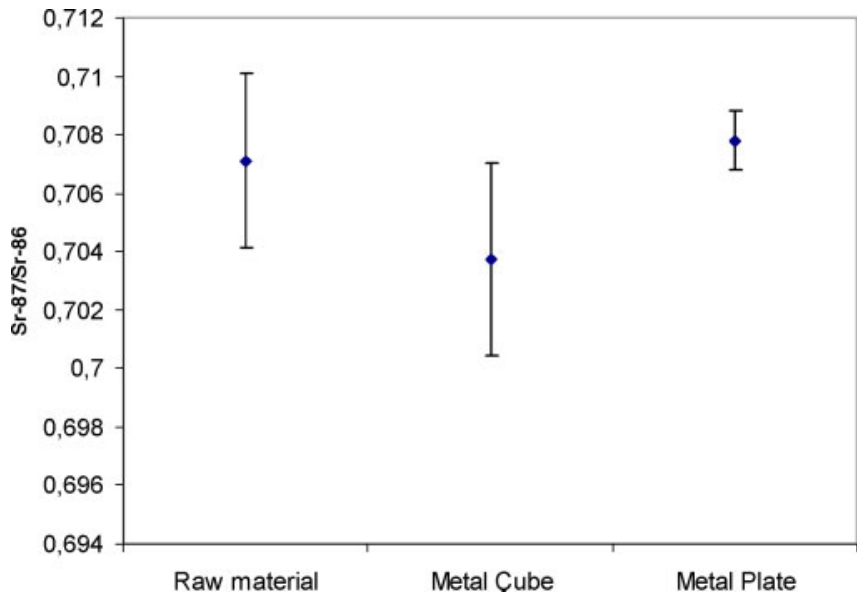

Fig. 5. $n\left({ }^{87} \mathrm{Sr}\right) / n\left({ }^{86} \mathrm{Sr}\right)$ isotope abundance ratio measured in the two uranium metal samples and compared to the likely source material, originating from Joachimstal. Error bars indicate the measurement uncertainty, expressed as combined standard uncertainty $U_{\mathrm{c}}$ with a coverage factor $k=2$.

from Joachimstal source material, not from Belgian Congo material.

\section{Sr isotope ratio measurements}

Another parameter that has proved to contribute to the origin assessment of natural uranium is the isotopic composition of strontium. The strontium isotope ratios vary over a wide range (between 0.7068-0.7606), and can possibly be used to distinguish samples of different origin as well as to apply for the verification of the source by the comparison with known samples [4]. As the $n\left({ }^{87} \mathrm{Sr}\right) / n\left({ }^{86} \mathrm{Sr}\right)$ ratio correlates with the age of the uranium deposit and/or its $\mathrm{Rb} / \mathrm{Sr}$ ratio, it may be expected that samples from deposits of similar age would have similar value in ore concentrates in distinct geological settings. However, the $n\left({ }^{87} \mathrm{Sr}\right) / n\left({ }^{86} \mathrm{Sr}\right)$ isotope ratio is also influenced by other factors such as the presence of minerals with high alkali metal content. The two samples were subjected to chemical separation using Sr Resin ${ }^{\mathrm{TM}}$ extraction chromatographic columns $(100-150 \mu \mathrm{m}$ particle size, supplied by Eichrom Technologies Inc., Darien, Illinois, USA). A NuPlasma ${ }^{\mathrm{TM}}$ (NU Instruments, Oxford, UK) double-focusing multi-collector inductively coupled plasma mass spectrometer (MC-ICP-MS), equipped with 11 Faraday collectors and 3 discrete dynode electrode multipliers was used for the $\mathrm{Sr}$ isotope measurements. The instrument was operated in low mass resolution mode. The samples were introduced into the plasma using a low-flow Teflon microconcentric nebulizer operated in a self-aspirating mode in combination with a desolvation unit (Aridus, CETAC Technologies Inc., Omaha, NE, USA). For instrument calibration and correction for instrumental isotopic fractionation SRM$987\left(\mathrm{SrCO}_{3}\right.$ isotope standard, National Institute of Standards and Technology, Gaithersburg, USA) was used.

As can be seen from Fig. 5, no significant difference between the two uranium metal samples was observed. Also the $n\left({ }^{87} \mathrm{Sr}\right) / n\left({ }^{86} \mathrm{Sr}\right)$ isotope abundance ratio for the Joachimstal ore as taken from literature [5] agrees very well. This finding confirms the indication that both uranium metal samples were produced from the same batch of starting material, which originates from Joachimstal.

\section{Age dating}

Another important parameter providing information on the authenticity of the two samples and on their production dates is the date of the last chemical purification of the uranium. The latter is referred to as the age of the material, as it describes the time elapsed since the last separation of uranium from its daughter nuclides. The method for age determination of uranium has been described in detail $[6,7]$. Aliquots of the respective uranium solution were spiked with a ${ }^{229} \mathrm{Th}$ spike. Uranium and thorium were then separated using a TEVA column (Eichrom Technologies Inc., Darien, Illinois, USA) using a specially developed procedure. The thorium fractions were measured by isotope dilution alpha spectrometry (ID-AS) and by isotope dilution mass spectrometry (ID-MS) using a MC-ICP-MS instrument. The ${ }^{234} \mathrm{U}$ was quantified by isotope dilution mass spectrometry using ${ }^{233} \mathrm{U}$ as spike isotope; the measurements were performed by thermal ionisation mass spectrometry (MAT 261, Thermo Finnigan, Bremen, Germany).

The age was calculated using the equation of radioactive decay and its derivatives:

$$
\begin{aligned}
& N=N_{0} \cdot \exp (-\lambda t) \\
& \frac{N_{\mathrm{U}-234}}{N_{\mathrm{Th}-230}}=\frac{\lambda_{\mathrm{U}-234}}{\lambda_{\mathrm{Th}-230}-\lambda_{\mathrm{U}-234}} \cdot\left(1-e^{\left(\lambda_{\mathrm{U}-234}-\lambda_{\mathrm{Th}-230}\right) \cdot t}\right) \\
& t=\frac{1}{\lambda_{\mathrm{U}-234}-\lambda_{\mathrm{Th}-230}} \cdot \ln \left(1-\frac{N_{\mathrm{Th}-230}}{N_{\mathrm{U}-234}} \cdot \frac{\lambda_{\mathrm{Th}-230}-\lambda_{\mathrm{U}-234}}{\lambda_{\mathrm{U}-234}}\right)
\end{aligned}
$$

where $N_{\mathrm{U}-234}$ is the number of ${ }^{234} \mathrm{U}$ atoms (at time $t$ ), $N_{\mathrm{Th}-230}$ is the number of ${ }^{230} \mathrm{Th}$ atoms (at time $t$ ), $\lambda_{\mathrm{U}-234}$ is the decay constant of ${ }^{234} \mathrm{U}, \lambda_{\mathrm{Th}-230}$ is the decay constant of ${ }^{230} \mathrm{Th}$, assuming that the number of ${ }^{230} \mathrm{Th}$ atoms at the time of the last separation $(t=0)$ was 0 .

We found for the "Heisenberg Cube" a separation date of September 1943 ( \pm 0.5 years) while the "Wirtz Plate" turned out to originate from an earlier separation date of August 1940 ( \pm 0.2 years). It should be noted, however, that the age determined by this method does not reflect the time of machining the uranium metal to the given geometry, nor does it describe the time of metal production (refined uranium oxide was reduced at $1100^{\circ} \mathrm{C}$ by means of calcium metal, with a calcium chloride flux, in an atmosphere of the inert gas argon, as described by Irving [8]). The date of the "Heisenberg Cube" separation is consistent with literature information on the change of reaction design, moving from an alternating layer approach to uranium cubes suspended in heavy water. Also the greater age of the plate as compared to the cube is consistent with historical information.

\section{Conclusion}

Investigative radiochemical methods are the backbone of nuclear forensic investigations. A number of characteristic parameters and signatures have been established, allowing the drawing of probable conclusions on the history of the material. This comprises the origin of the source material as well as the processes applied for material transformation. Multiple parameters generate a complex pattern which contains a wealth of information. The interpretation of the measured data and the comparison against values obtained on material of known history enable the establishment of the his- 
tory of material of unknown origin. This methodology can also be applied to questions related to illicit trafficking of nuclear material, to non-proliferation, to international safeguards and to environmental issues. In consequence, investigative radiochemistry serves as a deterrent against nuclear terrorism, proliferation or illegal waste dumping.

\section{References}

1. Richter, S., Goldberg, S. A.: Int. J. Mass Spectrom. 229, 181-197 (2003).

2. Varga, Z., Katona, R., Stefánka, Z., Wallenius, M., Mayer, K., Nicholl, A.: Talanta, doi:10.1016/j.talanta.2009.10.018 (2009).
3. Anders, E., Grevesse, N.: Geochim. Cosmochim. Acta 53(1), 197214 (1989).

4. Varga, Z., Wallenius, M., Mayer, K. , Keegan, E., Millet, S.: Anal. Chem. 81, 8327 (2009).

5. Möller, P., Dulski, P., Gerstenberger, H.: Appl. Geochem. 13(8), 975-994 (1998).

6. Wallenius, M., Morgenstern, A., Apostolidis, C., Mayer, K.: Anal. Bioanal. Chem. 374, 379-384 (2002).

7. Morgenstern, A.: Uranium age determination - Separation and analysis of Th-230 and Pa-231, International Conference on Advances in Destructive and Non-Destructive Analysis for Environmental Monitoring and Nuclear Forensics, Karlsruhe, Germany, 21-23 October (2002), http://www-pub.iaea.org/MTCD/publica tions/PDF/Pub1169_web.pdf

8. Irving, D.: The Virus House. Kimber, London (1967). 